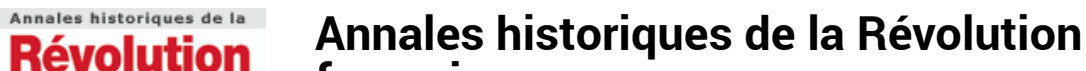

française française

344 | avril-juin 2006

La prise de parole publique des femmes

\section{Choix bibliographique}

Christine Fauré et Annie Geffroy

\section{(2) OpenEdition \\ Journals}

Édition électronique

URL : https://journals.openedition.org/ahrf/6453

DOI : 10.4000/ahrf.6453

ISSN : 1952-403X

\section{Éditeur :}

Armand Colin, Société des études robespierristes

\section{Édition imprimée}

Date de publication : 1 juin 2006

Pagination : 207-215

ISSN : 0003-4436

\section{Référence électronique}

Christine Fauré et Annie Geffroy, «Choix bibliographique », Annales historiques de la Révolution

française [En ligne], 344 | avril-juin 2006, mis en ligne le 01 juin 2009, consulté le 23 avril 2022. URL

http://journals.openedition.org/ahrf/6453 ; DOI : https://doi.org/10.4000/ahrf.6453

Ce document a été généré automatiquement le 23 avril 2022.

Tous droits réservés 


\title{
Choix bibliographique
}

\author{
Christine Fauré et Annie Geffroy
}

1 Articles, revues, ouvrages parus sur les femmes et la Révolution française depuis 1976. Pour la période antérieure, voir la bibliographie de Devance (1977).

\section{BIBLIOGRAPHIE}

ABERDAM Serge, « Droits de la femme et citoyenneté. Autour du séjour de Mary Wollstonecraft à Paris en 1793 ", dans Raymonde Monnier, Jacques Guilhaumou (dir.), Des notions-concepts en Révolution, Paris, SER, 2003, p. 123-148.

ABERDAM Serge, « Deux occasions de participation féminine en 1793 : le vote sur la Constitution et le partage des biens communaux », AHRF, n³39, janvier-mars 2005, p.17-34.

Albistur Maïté, ARMogathe Daniel, Histoire du féminisme français, du Moyen Age à nos jours, Paris, Éditions des femmes, 1977, 508 p.

Applewhite Harriet B., Levy Darline G., Johnson Mary D. (dir.), Women in Revolutionary Paris, Urbana, Chicago, London, University of Illinois Press, 1979, XI-325 p.

Applewhite Harriet B., LeVy Darline G. (dir.), Women and Politics in the Age of the Democratic Revolution, Ann Arbor, University of Michigan Press, 1990, 289 p.

ARBOUR Romeo, Dictionnaire des femmes libraires en France, 1470-1870, Genève, Droz, 2003, 750 p.

BADINTER Elisabeth (ed.), Paroles d'hommes 1790-1793, Paris, POL, 1989

BATTAGLINI Mario (ed.), Eleonora Fonseca Pimentel : il fascino di une donna impegnata fra letteratura e rivoluzione, Napoli, Procaccini, 1998, 170 p.

BENABOU Erica-Marie, La prostitution et la police des mœurs au 18e siècle, Paris, Perrin, 1987, 547 p. 
BERNEZ Marie-Odile, Une Anglaise défend la Révolution française, Paris, Éditions du CTSH, 2003, 482 p. (traduction et présentation de Mary Wollstonecraft, A Vindication of the Rights of Men, 1790).

Bessieres Yves, NIEDZWIECKI Patricia, Les femmes dans la Révolution française, 1789 : bibliographie, Bruxelles, Commission des Communautés européennes, les Cahiers de femmes d'Europe- 33, 1991, $88 \mathrm{p}$.

BlANC Olivier, Olympe de Gouges, Paris, Syros, 1981, 231 p. Édition revue et augmentée, Paris, Syros, 1989, 244 p.

Blanc Olivier (éd.), Olympe de Gouges, Écrits politiques, Paris, Coté-femmes, 1993, 2 vol.

Blanc Olivier, Marie-Olympe de Gouges. Une humaniste à la fin du 18e siècle, Editions René Viénet, 2003, $270 \mathrm{p}$.

BORDEAUX Michèle, «L'universalisme juridique et l'impasse de l'égalité », dans Marie-France Brive (dir.), Les femmes et la Révolution française, Toulouse, Presses Universitaires du Mirail, vol. 1, 1989 , p. 427-440

BRIVE Marie-France (dir.), Les femmes et la Révolution française, Toulouse, Presses Universitaires du Mirail, 3 vol, 1989-1991 (Actes du colloque de Toulouse, 1989)

BROUARD-ARENDS Isabelle (dir.), Lectrices d'Ancien Régime, Rennes, Presses Universitaires de Rennes, 2003, 720 p. (Actes du colloque de Rennes, 2002)

ButTAfuoco Annarita, «Eleonora Fonseca Pimentel : una donna nella rivoluzione », Donna, Woman, Femme, 3 aprile-giugno 1977, p. 51-92.

ButTAFUoco Annarita (dir.), Modi di essere : studi, reflessioni, interventi sulla cultura e la politica delle donne in onore di Elvira Badaracco, Bologna, EM, 1991.

CAPITAN Colette, La nature à l'ordre du jour 1789-1793, Paris, Kimé, 1993, 180 p.

COSANDEY Fanny, La reine de France : symbole et pouvoir, XV-XVIIIe siècle, Paris, Gallimard, 2000 , $414 \mathrm{p}$.

DAUPhin Cécile, FARGE Arlette (dir.), De la violence et des femmes, Paris, Albin Michel, 1997, 202 p.

DeRmenjian Geneviève, Guilhaumou Jacques, "Le crime héroïque de Charlotte Corday ", dans Geneviève Dermenjian, Jacques Guilhaumou, Martine Lapied (eds), Le Panthéon des femmes. Figures et représentations des héroïnes, Paris, Publisud, 2004, p. 149-159

Des sources pour l'histoire des femmes - guide, coordonné par Annick Tillier avec la collaboration d'Odile Faliu, Paris, BNF 2004, 204 p.

DESAN Suzanne, The Family on Trial in Revolutionary France, Berkeley, 2004, 456 p.

DESSERTINE Dominique, Divorcer à Lyon sous la Révolution et l'Empire, Lyon, Presses Universitaires de Lyon, 1981, $387 \mathrm{p}$.

DEVANCE Louis, « Le féminisme pendant la Révolution française », AHRF, 1977, n 229, p. 354-376.

Duby Georges, PERROT Michelle (dir.), Histoire des femmes en Occident, Paris, Plon, 5 vol., 1991-1992. Vol. 3, 16è-18è siècle (dir. DAVIES Nathalie et FARGE Arlette), 557 p. Vol. 4, 19è siècle (dir. FrAISSE Geneviève et PERROT Michelle), $627 \mathrm{p}$.

DuHET Paule-Marie, Les femmes et la Révolution, 1789-1794, Paris, Archives Gallimard - Julliard, 1971, 238 p. ; Paris, Archives Julliard, 1977. 
DuHET Paule-Marie, Cahiers de doléances des femmes en 1789, Paris, des Femmes, 1981, 230 p. ; 1989, XIII-218 p.

ELYADA Ouzi (ed.), Lettres bougrement patriotiques de la mère Duchesne, suivi du Journal des femmes : 1791, Paris, Éditions de Paris/ EDHIS, 1989, 195 p.

FARGE Arlette, KLAPISCH-ZUBER Christine (dir.), Madame ou Mademoiselle ? Itinéraires de la solitude féminine, 16e-18e s., Paris, Montalba, 1984, 302 p.

FAURÉ Christine, La démocratie sans les femmes. Essai sur le libéralisme en France, Paris, PUF, 1985, $264 \mathrm{p}$.

FAURÉ Christine, «Des droits de l'homme aux droits des femmes : une conversion intellectuelle difficile », dans Encyclopédie Politique et Historique des Femmes, p. 203-222.

FAURÉ Christine (dir.), Encyclopédie politique et historique des femmes. Europe, Amérique du Nord, Paris, PUF, 2e éd. 1997, 885 p.

FAURÉ Christine, « Une histoire des femmes au XVIIIe siècle par Louise de Kéralio ", Revue de la Bibliothèque Nationale de France, $\mathrm{n}^{\circ}$ 17, p. 61-64.

FAURÉ Christine, «L'offrande patriotique des femmes en 1789 », Revue de la Bibliothèque Nationale de France, $n^{\circ} 20,2005$, p. 69-73.

Les femmes et la Révolution française, bibliographie établie par Simone Blanc, Paris, Agence culturelle de Paris, 1989, 78 p.

Les femmes dans la Révolution française, Paris, EDHIS, 1982, 3 vol.

FRAISSE Geneviève, Muse de la raison. La démocratie exclusive et la différence des sexes, Aix, Alinéa, 1989, 224 p. ; réédition Folio/Gallimard, 1995.

FRAISSE Geneviève (éd. et préf.), Opinions de femmes, de la veille au lendemain de la Révolution française, Paris, Coté-femmes, 1989, $176 \mathrm{p}$.

FRAISSE Geneviève, La raison des femmes, Paris, Plon, 1992, 294 p.

FRAISSE Geneviève, Les deux gouvernements : la famille et la cité, Paris, Folio Essais, 2000, 224 p.

Gefrroy Annie, «Leçons sur l'(in)égalité des sexes (Frantext 1789-1820) » dans Pierre Fiala (dir.), In/égalité/s. Usages lexicaux et variations discursives (18e-20e siècles), Paris, L'Harmattan, 1999, p. 43-69.

GEFFroy Annie, « La citoyenneté sexiste en France, 1789-1946 : les mots pour la dire », dans Claude Fiévet (dir.), Invention et réinvention de la citoyenneté, Pau, Éditions Joëlle Sampy, 2000, p. 213-223 (Actes du colloque international de Pau, 1998).

GODINEAU Dominique, Citoyennes tricoteuses : les femmes du peuple à Paris pendant la Révolution française, Aix, Alinea, 1988, 417 p. ; Paris, Perrin, 2004, 416 p.

GODINEAU Dominique, Les femmes dans la société française, 16e-18e siècle, Paris, Armand Colin, 2003, $254 \mathrm{p}$.

GODINEAU Dominique, « Filles de la liberté et citoyennes révolutionnaires », Histoire des femmes en Occident, 19e siècle, Paris, Plon, 1991, vol. 4 (sous la dir. Geneviève Fraisse et Michelle Perrot), p. 27-42

GODINEAu Dominique, «Travail et politique à Paris pendant la Révolution : l'exemple des ateliers municipaux », AHRF, 1986, p. 35-49. 
GUIBERT-SLEDZIEWSKI Elisabeth, Révolutions du sujet, Paris, Méridiens Klincksieck, 1989, 318 p.

GUIBERT-SLEDZIEWSKI Elisabeth, « La femme, objet de la Révolution », AHRF, 1987, p. 1-16.

GUIBERT-SLEDZIEWSKI Elisabeth, « Révolution française, le tournant », Histoire des femmes en Occident, 19e siècle, Paris, Plon, 1991, vol. 4 (sous la dir. Geneviève Fraisse et Michelle Perrot), p. 43-56.

Grubitzsch Helga, BocкHolt Roswitha, Théroigne de Méricourt, die Amazone der Greiheit, Pfaffenweiler, Centaurus, 1991.

GUIBERT Noëlle, RAZGonNiKov Jacqueline, Le journal de la Comédie française, 1787-1799. La comédie aux trois couleurs, Antony, Sides, 1989, 389 p.

GuILHAUMOU Jacques, LAPIED Martine, « L'action politique des femmes pendant la Révolution française ", dans Christine Fauré (dir), Encyclopédie politique et historique des femmes, Paris, PUF, 1997, p. 139-168.

GuilhaUmou Jacques, LAPIED Martine, « Les femmes dans les archives des comités de surveillance des Bouches-du-Rhône », dans Geneviève Dermenjian, Jacques Guilhaumou, Martine Lapied (eds), Femmes entre ombre et lumière. Recherches sur la visibilité sociale (XVIe-XXe siècles), Paris, Publisud, 2000 , p. 247-258.

GuilHaUmou Jacques, « L'exclusion des femmes du savoir politique pendant la Révolution française ", dans Luc Capdevila, Sophie Cassagnes, Martine Cocaud, Dominique Godineau et al, Le genre face aux mutations. Masculin et féminin du Moyen Âge à nos jours, Rennes, Presses Universitaires de Rennes, 2003, p. 265-275.

HARTEN Elke, HARTEN Hans-Christian, Femmes, culture et révolution, Paris, Des femmes/Antoinette Fouque, 1989, 590 p. (trad. de l'allemand 1988).

HEsSE Carla, «French Women in Print, 1750-1800 : an Essay in Historical Bibliography », dans Haydn T. Mason (ed.), The Darnton Debate : Books and Revolution in the Eighteen Century, Oxford, Voltaire Foundation, 1998, p. 65-82.

HESSE Carla, The Other Enlightenment. How French Women Became Modern, Princeton, Princeton University Press, 2001, X-234 p.

HEUER Jennifer, « Family Bonds and Female Citizenship : émigré Women under the Directory », dans Howard G. Brown et Judith A. Miller (dir.), Taking Liberties : Problems of a New Order from the French Revolution to Napoleon, New York, 2002, Manchester University Press, p. 51-70.

HÖFER Annette, KEILHAUER Annette, Femme, fasc. 16 du Handbuch politisch-sozialer grundbegriffe im Frankreich, 1680-1820, Munchen, Oldenburg, 1996, 84 p.

HUFTON Olwen H., Women and the Limits of Citizenship in the French Revolution, Toronto, University of Toronto Press, 1992, XIII-201 p.

Hunt Lynn, Politics, Culture and Class in the French Revolution, Berkeley, Los Angeles, London, University of California press, 1984, XV-251.

HuNT Lynn, Le roman familial de la Révolution française, Albin Michel, 1995, 264 p. (trad. de The Family Romance ..., 1992).

KALE Steven, French Salons, High Society and Political Sociability from the Old Regime to the Revolution of 1848, Baltimore/London, Johns Hopkins University Press, 2005.

KRUMENACKER Yves (dir.), Religieux et religieuses pendant la Révolution, 1770-1820, Lyon, Profac, 1995, 2 vol (Actes du colloque de Lyon, 1992). 
LAMBERT Karine, « Genre et transmission du patrimoine face aux mutations “juridiques” en Provence orientale (1775-1798)», dans Luc Capdevila, Sophie Cassagnes, Martine Cocaud, Dominique Godineau et al., Le genre face aux mutations. Masculin et féminin du Moyen Âge à nos jours, Rennes, Presses Universitaires de Rennes, 2003, p. 233-242.

LANDES Joan B., Vizualizing the Nation : Gender, Representation and Revolution in 18th Century France, Ithaca, Londres, Cornell University Press, 2001, XIII-254 p.

LANDES Joan B., Women and the Public Sphere in the Age of the French Revolution, Ithaca, Cornell University Press, 1988, XI-275 p.

LAPIED Martine et RICHARD Éliane (dir.), « Femmes et politiques en Provence, XVIIIe-XXe siècles », Provence historique, $\mathrm{n}^{\circ} 186,1996$.

LAPIED Martine, «La fanatique contre-révolutionnaire, réalité ou représentation ? », dans Capdevila L., Cassagnes S., Cocaud M., Godineau D. et al. Le genre face aux mutations. Masculin et féminin du Moyen Âge à nos jours, Rennes, Presses Universitaires de Rennes, 2003, p. 255-264. LAPIED Martine et Peyrard Christine (dir.), « Histoire des femmes » (articles de Dominique Godineau, Lynn Hunt, Martine Lapied, Laura Pisano), dans La Révolution française au carrefour des recherches, Aix-en-Provence, Publications de l'Université de Provence, 2003, p. 281-321.

LAPIED Martine, « Histoire du Genre en révolution », dans Jean-Clément Martin (dir.), La Révolution à l'œuvre. Perspectives actuelles dans l'histoire de la Révolution française, Rennes, Presses Universitaires de Rennes, 2005, p. 77-87.

Lejeune Philippe, Lucile Desmoulins, Journal, 1788-1793, Paris, Editions des Cendres, 1995, 164 p.

LHOTE Jean, Le divorce à Metz et en Moselle sous la Révolution et l'Empire : une anticipation sociale, Metz, J. Lhote, 1981, 48 p.

MARAND-FOUQUET Catherine, La femme au temps de la Révolution, Paris, Stock/ Laurence Pernoud, 1989, $417 \mathrm{p}$.

MARTIN Jean-Clément, « Femmes et guerre civile, l'exemple de la Vendée, 1793-1796 », Clio/ Histoire, femmes et sociétés, Toulouse, $\mathrm{n}^{\circ}$ 5, 1997, « Guerres civiles » p. 97-115.

MORIN-ROTUREAU Eveline (dir.), 1789-1799, combats de femmes : les révolutionnaires excluent les citoyennes, Paris, Autrement, 2003, 244 p.

MAZA Sarah, Vies privées, affaires publiques. Les causes célèbres de la France prérévolutionnaire, Paris, Fayard, 1997 (trad. de Private Lives..., Berkeley, University of California Press, 1993).

MAZEL Geneviève, « Louise de Kéralio et Pierre-François Robert, précurseurs de l'idée républicaine », Bulletin de la Société d'Histoire de Paris et de l'Ile-de-France, 1989, p. 163-237.

Menant Sylvain (dir.), «Femmes des Lumières », Dix-huitième siècle, n 36, 2004, 375 p.

OfFEN Karen, European Feminisms. A Political History, 1700-1950, Stanford, Stanford University Press, $2000,554 \mathrm{p}$.

OUTRAm Dorinda, The Body and the French Revolution: Sex, Class and Political Culture, New Haven, Yale university press, 1989, IX-197 p.

Ozouf Mona, Les mots des femmes. Essai sur la singularité française, Paris, Fayard, 1995, 400 p. (Rééd + postface Gallimard/Tel, 1999).

Petersen Suzanne, Frauen in der Franzosische Revolution, Berlin, Akademie-Verlag, 1987. 
PIGEAUD Jackie, Théroigne de Méricourt. La lettre-mélancolie, Lagrasse, Verdier/L'éther vague, 2005, $50 \mathrm{p}$.

PISANO Laura, VEAUVY Christine (dir.), Parole inascoltate. Le donne e la costruzione dello Stato-nazione in Italia e in Francia (1789-1860). Testi e documenti, prefazione di Ginevra Conti Odorisio, Roma, Editori Riuniti, 1994 ; Paroles oubliées: les femmes et la construction de l'État nation en France et en Italie, 1789-1860, préface de Michelle Perrot, Paris, A. Colin, 1997, XI-339 p.

Proctor Candice, Women, Equality and the French Revolution, New York, Greenwood press, 1990, XIII-210 p.

RAGAN Jr. Bryant T., WILlIAMS Elizabeth A., Re-creating Authority in Revolutionary France, NewBrunswick, Rutgers University press, 1992, XII-235 p.

ReNDALL Jane, The Origins of Modern Feminism. Women in Britain, France and the United States, London, MacMillan, 1985.

RODERICK Phillips, Family Breakdown in Late Eighteen-Century France : Divorces in Rouen, 1792-1803, Oxford, Clarendon Press, 1980, VIII-244 p.

Rosa Annette, Citoyennes, les femmes et la Révolution française ; préface de Claude Mazauric, postface de Elisabeth Sledziewski, Paris, Messidor, 1988, 253 p.

ROUDINESCo Elisabeth, Théroigne de Méricourt. Une femme mélancolique sous la Révolution, Paris, Seuil, 1989,316 p.

SANTOS Teresa, PEREIRA Sara Marques (dir.), Leonor da Fonseca Pimentel : a portuguesa de Nápoles (1752-1799), Lisboa, Livros Horizonte, 2001, 231 p.

ScoTT Joan W., La citoyenne paradoxale. Les féministes françaises et les droits de l'homme, Albin Michel, 1998, 290 p. (trad. de Only paradoxes to offer..., Harvard University press, 1996).

SIESS Jurgen, « Un discours politique au féminin. Le projet d'Olympe de Gouges », Mots, n 78, juillet 2005, p. 9-21.

SOBOUL Albert, MONNIER Raymonde, Répertoire du personnel sectionnaire parisien en l'an II, Paris, Publications de la Sorbonne, 1985, 564 p. (199 notices sur des militantes, par section.

STEINER Gehrard, «L'engagement et le destin des “jacobines” de Mayence », AHRF, 1984, p. 124-154.

THÉBAUD Françoise, Écrire l'histoire des femmes, Paris, ENS Editions, 1998, 218 p.

THÉRY Irène, BIET Christian (eds), La famille, la loi, l'État, de la Révolution au Code civil, Paris, Imprimerie nationale/ Centre Georges Pompidou, 1989, XXIV-534 p.

VAN DIJK Suzanna, Traces de femmes : présence féminine dans le journalisme français du 18e siècle, Amsterdam/Maarsen, APA-Holland University press, 1988, XIII-330 p.

VERJUS Anne, Le cens de la famille. Les femmes et le vote, 1789-1848, Paris, Belin, 2002, 256 p.

VIENNOT Eliane (dir.), La démocratie «à la française » ou les femmes indésirables, Paris, Publications de l'Université Paris-7, 1996, 288 p. (Actes du colloque de Paris, 1993).

VIGNI Francesca, « Les aspirations féministes dans les loges d'adoption en France », Dix-huitième siècle, 19, 1987, p. 211-220.

WHALEY Leigh, « Partners in Revolution : Louise de Kéralio and François Robert, editors of the Mercure national, 1789-1791 », dans Malcolm Crook, William Doyle, Alan Forrest (eds).

Enlightenment and Revolution. Essays in Honour of Norman Hampson, Ashgate, 2004, p. 114-131. 
WinN Colette, KUIZENGA Donna, Women Writers in Pre-Revolutionary France : Strategies of Emancipation, New-York/ Londres, Garland, 1997, XXX-454 p.

WOLLSTONECRAFT Mary, Défense des droits de la femme, préf. et trad. de Marie-Françoise Cachin, Paris, Payot, 1975, 242 p. 